\title{
Incidência de doenças no pós-parto de primíparas da raça Holandesa alimentadas com diferentes fontes energéticas durante o período de transição
}

\author{
[Disease incidence in the postpartum period of primiparous Holstein cows fed diets with different energetic sources \\ during the transition period]
}

\author{
M.A.T. Artunduaga ${ }^{1}$, S.G. Coelho ${ }^{2}$, A.M.Q. Lana $^{2}$, B.G. Campos ${ }^{1}$, R.B. Reis ${ }^{2}$, \\ H.M. Saturnino ${ }^{2}$, R.V.S. Fortes ${ }^{3}$, H.N. Costa ${ }^{3}$ \\ ${ }^{1}$ Facultad de Ciencias Agropecuarias. Universidad de La Salle. Bogotá, Colômbia \\ ${ }^{2}$ Escola de Veterinária - UFMG \\ Caixa Postal 567 \\ 30123-970 - Belo Horizonte, MG \\ ${ }^{3}$ Rehagro
}

\section{RESUMO}

Avaliaram-se o efeito da adição de diferentes fontes energéticas na dieta de vacas primíparas leiteiras, durante o período de transição, sobre a produção e a composição do leite, as ocorrências clínicas no pósparto e sua relação com as concentrações plasmáticas de ácidos graxos não esterificados (AGNE). Foram utilizadas 50 vacas primíparas da raça Holandesa no período de 28 dias antes da data prevista do parto até o $21^{0}$ dia pós-parto. Os animais foram designados aos grupos: controle, Megalac- E®, soja tostada (ST) ou propileno glicol (PG). Avaliações clínicas foram realizadas todos os dias. Amostras de sangue foram coletadas no pré e pós parto, antes da primeira alimentação do dia, para avaliação de AGNE. Os grupos Megalac- ${ }^{\circledR}$ e propileno glicol apresentaram maior volume de leite corrigido para 3,5\% de gordura (LCG 3,5\%) em relação ao grupo soja tostada $(\mathrm{P}=0,05)$. Nos grupos controle, Megalac- E® e soja tostada, os valores de AGNE aumentaram até o parto, seguidos de queda após o parto. Essa queda foi menos acentuada no grupo-controle, que apresentou equação de regressão quadrática, estando associado à maior incidência de afecções no pós-parto. O grupo Megalac-E® apresentou comportamento cúbico, o soja tostada hiperbólico, e o propileno glicol quadrático.

Palavras-chave: aditivo gliconeogênico, gordura, periparto

\begin{abstract}
The purpose of this trial was to evaluate the effect of different energy sources added to the diet over milk production and composition, disease incidence during postpartum period and its relationship to NEFA concentrations during the transition period. Fifty primiparous Holstein dairy cows were used from 28 days before the expected calving date until 21 days postpartum. Cows were randomly distributed in four groups, being: control, Megalac- E®, toasted soybean (TS) and propylene glycol (PG). Clinical evaluations were performed during all experimental days. Blood samples were collected in pre and postpartum periods, before the first feeding of sampling day in order to determine NEFA prolife. Megalac- E® and propylene glicol groups showed greater milk production corrected to 3.5\% fat compared to group toasted soybean $(P=0.05)$. In control groups, Megalac-E ${ }^{\circledR}$ and toasted soybean values of NEFA increased until delivery and then decreased. This decrease was less pronounced in the control group that showed a quadratic regression equation associated with higher incidence of postpartum disorders. The Megalac-E® group showed a cubic model, the toasted soybean hyperbolic and propylene glycol quadratic equations.
\end{abstract}

Keywords: dairy cow, fat supplementation, glyconeogenic additive, peripartum

Recebido em 10 de maio de 2010

Aceito em 14 de abril de 2011

*Autor para correspondência (corresponding author)

E-mail: sandragesteiracoelho@gmail.com 


\section{INTRODUÇÃo}

O período de transição compreende as três semanas pré e pós-parto (Grummer, 1995; Drackley, 1999) e é considerado crítico para a saúde e a produtividade, pois o animal passa do estado fisiológico gestante não lactante para lactante. Essa transição é marcada por grandes mudanças endócrinas e metabólicas. Na fase préparto, os estoques maternos de nutrientes são direcionados para o crescimento e sobrevivência fetal, a formação do colostro e a preparação da glândula mamária para o início da lactação, e na fase pós-parto, o metabolismo materno está inteiramente voltado para a síntese de leite (Drackley, 1999).

De acordo com Drackley et al. (2005), as exigências de energia líquida, em primíparas, dois dias antes do parto, mudam de 58,5 para 105,0 (MJ/dia) dois dias após o parto. Essa mudança brusca nas exigências nutricionais não é compensada pela ingestão de matéria seca, a qual é reconhecida por estar diminuída no período de transição. Assim, tanto no pré quanto no pós-parto, observa-se desequilíbrio entre as necessidades do animal e o consumo de nutrientes, fazendo com que as necessidades energéticas e proteicas sejam supridas pela mobilização de reservas corporais (Ingvartsen e Andersen, 2000), o que leva o animal a apresentar o quadro conhecido como balanço energético negativo (BEN), que é normalmente observado durante as últimas três semanas da gestação e as primeiras semanas da lactação.

O BEN é um processo de adaptação complexo que inclui ajuste integrado entre processos, tais como gliconeogênese, glicogenólise, lipólise, metabolismo de proteínas, entre outros, podendo variar de intensidade entre os animais (expressão genética). Esses ajustes têm o objetivo de adaptar o animal o mais rápido possível ao novo estado fisiológico. No entanto, o excessivo BEN predispõe o animal à manifestação de doenças de origem metabólica, como hipocalcemia, acetonemia clínica e subclínica, hipomagnesemia e afecções do pós-parto imediato, como o deslocamento de abomaso, a retenção de placenta, a metrite e a mastite, entre outras (Drackley, 1999).

A mobilização das reservas corporais causadas pelo BEN faz com que as concentrações plasmáticas de AGNE aumentem à medida que se aproxima o parto, sendo os AGNE considerados um biomarcador de balanço energético negativo, uma vez que indicam quebra de gordura corporal (lipólise), em resposta à crescente demanda de energia onde 0 suprimento de glicose é insuficiente para satisfazer as necessidades energéticas. LeBlanc et al. (2005) demonstraram que a alta concentração de AGNE antes do parto está fortemente relacionada a afecções no pós-parto imediato. Para Ospina et al. (2010), as concentrações de AGNE de $0,29 \mathrm{mEq} / \mathrm{L}$ no préparto e $0,57 \mathrm{mEq} / \mathrm{L}$ no pós-parto são o limite crítico para o desenvolvimento de doenças no período de transição. Segundo Leblanc et al. (2006), aproximadamente $75 \%$ das doenças de vacas de leite tipicamente ocorrem no primeiro mês após o parto.

Dessa forma, o objetivo deste trabalho foi avaliar as ocorrências clínicas após o parto e sua relação com a concentração sérica de AGNE em vacas primíparas que receberam diferentes fontes energéticas durante o período de transição.

\section{MATERIAL E MÉTODOS}

Todos os procedimentos experimentais foram aprovados pelo Comitê de Ética em Experimentação Animal da Universidade Federal de Minas Gerais, certificado 40/2007.

O experimento foi realizado em uma fazenda particular durante o período de março a julho de 2007. Foram utilizadas 50 primíparas da raça Holandesa no período de 28 dias antes da data prevista para o parto até o $21^{\circ}$ dia pós-parto. Os animais foram alojados em quatro lotes separados, dentro de um free stall, com área específica para a parição, que era liberada somente momentos antes do parto. Os animais foram distribuídos aleatoriamente aos grupos experimentais de acordo com a fonte energética suplementar. Os do grupo-controle receberam dieta base no pré e pós-parto; os do grupo Megalac- E® (Arm \& Hammer, QGN) receberam sais de cálcio de ácidos graxos da soja $100 \mathrm{~g}$ no pré-parto e $250 \mathrm{~g}$ no pós-parto; os do grupo soja tostada receberam dieta base suplementada com $400 \mathrm{~g}$ de soja tostada no préparto e $800 \mathrm{~g}$ no pós-parto, e os do grupo propileno glicol dieta base suplementada com $300 \mathrm{~mL}$ de propileno glicol no pré e pós parto. As 
fontes energéticas foram fornecidas uma vez ao dia, antes da primeira alimentação, misturadas a $500 \mathrm{~g}$ de milho moído, após individualização dos animais, segundo Artunduaga et al. (2010). As dietas foram formuladas de acordo com as recomendações do NRC (Nutrient..., 2001), para cada fase do período de transição (Tab. 1 e Tab. 2).

Tabela 1. Ingredientes e composição nutricional das dietas experimentais usadas no pré-parto de vacas de leite, de acordo com os tratamentos

\begin{tabular}{|c|c|c|c|c|}
\hline & Controle & Megalac- E®* & Soja tostada & Propileno glicol \\
\hline \multicolumn{5}{|l|}{ Ingrediente, $\mathrm{kg}$} \\
\hline Silagem de milho & 24,0 & 24,0 & 24,0 & 24,0 \\
\hline Milho moído & 1,0 & 0,5 & 0,5 & 0,5 \\
\hline Farelo de soja & 1,6 & 1,6 & 1,4 & 1,6 \\
\hline Soja tostada & ----- & ----- & 0,4 & ----- \\
\hline Megalac - E* & ----- & 0,1 & ----- & ----- \\
\hline Propileno-glicol (mL) & ----- & ----- & ----- & 300 \\
\hline $\begin{array}{c}\text { Premix min-vit pré-parto } \\
\text { (Nutron Pré-parto } 570 \text { CI Biotina) }\end{array}$ & 0,4 & 0,4 & 0,4 & 0,4 \\
\hline \multicolumn{5}{|l|}{ Nutriente, \% da MS } \\
\hline Matéria seca & 42,1 & 42,1 & 42,2 & 42,1 \\
\hline Proteína bruta & 14,6 & 14,8 & 14,9 & 14,9 \\
\hline Fibra em detergente neutro & 41,0 & 42,0 & 42,5 & 42,5 \\
\hline Fibra em detergente ácido & 23,6 & 24,4 & 24,7 & 24,6 \\
\hline Extrato etéreo & 3,5 & 3,5 & 3,5 & 3,5 \\
\hline $\mathrm{Ca}$ & 0,7 & 0,7 & 0,7 & 0,7 \\
\hline $\mathrm{P}$ & 0,4 & 0,4 & 0,4 & 0,4 \\
\hline Energia líquida (Mcal/kg) & 1,5 & 1,5 & 1,5 & 1,5 \\
\hline
\end{tabular}

*Megalac- E® sais de cálcio de ácidos graxos poli-insaturados; composição: extrato etéreo 85\%; ácido linoleico (C18:2): 45\%; ácido linolênico (C18:3): 6\%.

${ }^{1}$ Premix min-vit pré-parto, composição por kg: Ca: 5\%; P: 1\%; Mg: 1\%; Na: 1\%; Cl: 1,15\%; S: 0,8\%; Co: 39,3ppm; Cu: 660ppm; Fe: 775ppm; I: 19,5ppm; Mn: 1630ppm; Se: 8,98 ppm; Zn: 2368ppm; Vit. A: 260KIU; Vit. D: 35KIU; Vit. E: 2600UI; Biotina: 100ppm.

As análises bromatológicas das dietas foram realizadas para matéria seca, proteína bruta, fibra insolúvel em detergente neutro (FDN), fibra insolúvel em detergente ácido (FDA), cálcio e fósforo segundo a AOAC (Official..., 1980). O fornecimento das fontes energéticas foi iniciado 28 dias antes da data prevista para o parto e mantido até o $21^{\underline{0}}$ dia de lactação, a partir do qual todos os animais passaram a receber alimentação em quantidade suficiente para atender às necessidades nutricionais da lactação segundo o NRC (Nutrient..., 2001).
A produção de leite foi mensurada nos dias 10, 20, 30 e 40 da lactação. Nestes mesmos dias, foram obtidas amostras para determinação da composição do leite. As amostras foram refrigeradas em recipientes plásticos a $4^{\circ} \mathrm{C}$ com bromopol (2-bromo 2-nitropropano 1,3-diol), na relação de $10 \mathrm{mg}$ de bromopol para $50 \mathrm{~mL}$ de leite. As amostras foram enviadas para análise da composição química utilizando-se o método de raios infravermelhos proximais, sendo usado o aparelho Bentley 2000 (Bentley Instruments, Chaska, EUA). 
Incidência de doenças no pós-parto...

Tabela 2. Ingredientes e composição nutricional das dietas experimentais usadas no pós-parto de vacas de leite

\begin{tabular}{|c|c|c|c|c|}
\hline & \multicolumn{4}{|c|}{ Grupo } \\
\hline & Controle & Megalac- E®* & Soja Tostada & Propileno glicol \\
\hline \multicolumn{5}{|l|}{ Ingrediente, $\mathrm{kg}$} \\
\hline Silagem de milho & 32,0 & 32,0 & 32,0 & 32,0 \\
\hline Milho moído & 4,5 & 3,5 & 3,5 & 4,5 \\
\hline Farelo de soja & 4,2 & 4,5 & 3,9 & 4,2 \\
\hline Soja tostada & ----- & ---- & 0,8 & ----- \\
\hline Megalac - E* & ----- & 0,25 & ----- & ----- \\
\hline Propileno-glicol (mL) & ----- & ----- & ----- & 300 \\
\hline Bicarbonato de Sódio & 0,12 & 0,12 & 0,12 & 0,12 \\
\hline Fosfato bicálcico & 0,12 & 0,12 & 0,12 & 0,12 \\
\hline Calcáreo & 0,14 & 0,14 & 0,14 & 0,14 \\
\hline Óxido de magnésio & 0,04 & 0,04 & 0,04 & 0,04 \\
\hline $\begin{array}{l}\text { Premix min-vit pós-parto } \\
\text { (NutronPhos } 150 \text { ADE) }\end{array}$ & 0,08 & 0,08 & 0,08 & 0,08 \\
\hline \multicolumn{5}{|l|}{ Nutriente, \% da MS } \\
\hline Matéria seca & 40,1 & 40,1 & 40,1 & 40,1 \\
\hline Proteína bruta & 16,9 & 17,6 & 17,5 & 16,9 \\
\hline Fibra em detergente neutro & 33,9 & 34,4 & 35,3 & 33,9 \\
\hline Fibra em detergente ácido & 19,2 & 19,7 & 20,2 & 19,2 \\
\hline Extrato etéreo & 3,7 & 3,8 & 3,7 & 3,6 \\
\hline $\mathrm{Ca}$ & 1,1 & 1,1 & 1,1 & 1,1 \\
\hline $\mathrm{P}$ & 0,5 & 0,5 & 0,5 & 0,5 \\
\hline Energia líquida (Mcal/kg) & 1,7 & 1,7 & 1,7 & 1,7 \\
\hline
\end{tabular}

* Megalac- E® sais de cálcio de ácidos graxos poli-insaturados

${ }^{2}$ Premix min-vit pós-parto, composição por kg: Ca: 21\%; P 15\%; Mg:3\%; S: 3\%; Co: 100ppm; Cu: 3000ppm; I: 180ppm; Mn: 5000ppm; Se: 80ppm; Zn: 12000ppm; Vit. A: 1000KIU; Vit. D: 250KIU; Vit. E: 3250UI.

A produção de leite corrigida para $3,5 \%$ de gordura (LCG 3,5\%) foi obtida pela equação citada por Gravet (1987).

Amostras de sangue para determinação das concentrações plasmáticas de AGNE foram coletadas via punção da veia ou artéria coccígea em tubos de $5 \mathrm{~mL}$ com vácuo e EDTA antes do fornecimento das fontes energéticas. As amostras foram coletadas diariamente a partir de 15 dias antes da data prevista para o parto, no dia do parto e sete, 14, e 21 dias após o parto, para avaliação de AGNE. Apenas as amostras que correspondiam aos dias 10 e cinco antes do parto, ao dia do parto e aos dias sete, 14 e 21 após o parto foram analisadas. As concentrações plasmáticas de AGNE foram determinadas utilizando-se kit enzimático (Randox do Brasil). Avaliações clínicas foram realizadas todos os dias do experimento, e os casos de retenção de placenta, metrite, deslocamento de abomaso, diarreia, doença respiratória foram registrados em fichas individuais e tratados de acordo com a doença.

A homocedasticidade e a normalidade dos dados foram testadas pelos testes de Bartlett e Lilliefors, sendo os valores de AGNE transformados logaritmicamente. A produção e composição do leite e as concentrações de AGNE foram analisadas utilizando-se o delineamento inteiramente ao acaso em arranjo 
em parcelas subdivididas, sendo os grupos as parcelas, e o tempo as subparcelas. Para testar as diferenças entre as médias, o teste estatístico utilizado foi o SNK a 5\%. A concentração de AGNE ao longo do período experimental foi avaliada por modelos de regressão.

\section{RESULTADOS E DISCUSSÃO}

Não foi observada diferença para volume de leite entre os diferentes grupos $(\mathrm{P}=0,07)$. Os grupos Megalac-E e propileno glicol apresentaram maior volume de leite corrigido para 3,5\% de gordura (LCG 3,5\%) em relação ao grupo soja tostada $(\mathrm{P}=0,05)$. Não foi observado efeito dos aditivos na composição do leite para os teores de gordura e proteína $(\mathrm{P}>0,05)$, porém o grupo propileno glicol apresentou maior teor de lactose quando comparado com os demais grupos $(\mathrm{P}=0,01)$. O propileno glicol é um aditivo gliconeogênico e, dessa forma, pode ter fornecido mais glicose para glândula mamária, alterando os valores de lactose.

Tabela 3. Média e desvio-padrão da produção de leite, da LCG 3,5\% e composição do leite nos primeiros 40 dias de lactação, de vacas primíparas da raça Holandesa alimentadas com diferentes fontes energéticas durante o período de transição

\begin{tabular}{lcccccc}
\multicolumn{1}{c}{ Variável } & Controle & Megalac-E & Soja tostada & $\begin{array}{c}\text { Propileno } \\
\text { glicol }\end{array}$ & $\mathrm{P}$ & $\mathrm{CV}(\%)$ \\
\hline Produção de leite (kg) & $23,4 \pm 7,2$ & $24,8 \pm 4,6$ & $21,7 \pm 5,6$ & $24,4 \pm 5,8$ & 0,07 & 24,9 \\
LCG 3,5\% (kg) & $23,3 \pm 7,0 \mathrm{AB}$ & $24,9 \pm 4,9^{\mathrm{a}}$ & $21,7 \pm 5,7 \mathrm{~B}$ & $24,6 \pm 5,3 \mathrm{~A}$ & 0,05 & 24,4 \\
Proteína (\%) & $3,1 \pm 0,2$ & $3,0 \pm 0,2$ & $3,1 \pm 0,2$ & $3,0 \pm 0,2$ & 0,98 & 6,5 \\
Gordura (\%) & $3,5 \pm 0,4$ & $3,5 \pm 0,4$ & $3,5 \pm 0,4$ & $3,6 \pm 0,5$ & 0,98 & 11,9 \\
Lactose (\%) & $4,5 \pm 0,2 \mathrm{~B}$ & $4,5 \pm 0,2 \mathrm{~B}$ & $4,5 \pm 0,2 \mathrm{~B}$ & $4,6 \pm 0,2 \mathrm{~A}$ & 0,01 & 4,1 \\
\hline
\end{tabular}

Médias seguidas de letras distintas na linha diferem entre si pelo teste SNK $(\mathrm{P}<0,05)$.

MEGALAC- E®: sais de cálcio de ácidos graxos poli-insaturados.

LCG 3,5\%: leite corrigido para 3,5\% de gordura.

$\mathrm{P}$ : valor de probabilidade na ANOVA.

Apesar de o Megalac-E e de a soja tostada serem considerados fontes de gordura inerte ou potencialmente inerte no rúmen, e por apresentarem perfis de ácidos graxos similares, uma vez que são produtos compostos à base de soja, os dois grupos tiveram respostas produtivas diferentes (LCG 3,5\% P<0,05). Essa diferença pode ter sido causada pela forma de fornecimento da soja tostada uma vez ao dia e separada da dieta. Esse modo de fornecimento pode ter interferido negativamente nos processos de fermentação ruminal, visto que os ácidos graxos poli-insaturados podem ter sido liberados rapidamente no rúmen, afetando a microbiota e interferindo nos processos de degradabilidade da fibra e na taxa de passagem que levariam ao menor consumo de alimentos e menor aporte de substratos para produção de leite.

Os grupos controle e soja tostada apresentaram o maior número de ocorrências clínicas, (Tab. 4). Sabe-se que, durante o período de transição, acontece imunossupressão (Kehrli et al., 1989), e esta redução na habilidade do sistema imune em responder a processos infecciosos é provavelmente responsável pela alta incidência de mastite e metrite no pós-parto imediato (Drackley et al., 2005). As razões para a imunossupressão durante o período de transição não têm sido bem esclarecidas, mas evidências sugerem que o balanço energético negativo poderia ser o fator que afeta a resposta imunológica dos animais neste período (Goff, 1999).

As concentrações de AGNE aumentaram à medida que se aproximava o parto em todos os grupos (Fig. 1). Os grupos controle, Megalac-E e soja tostada apresentaram no dia -5 pré parto concentrações de AGNE acima de $0,29 \mathrm{mEq} / \mathrm{L}$, que, segundo Ospina et al. (2010), indicam riscos de desenvolvimento de deslocamento de abomaso, cetose, retenção de placenta e metrite. Com exceção de cetose, que não foi avaliada neste estudo, os três grupos tiveram animais com manifestações dessas afecções. No entanto, apesar de as concentrações de AGNE no grupo propileno glicol não terem atingido esses valores no pré-parto, esse grupo também apresentou animais com metrite e deslocamento de abomaso. 
Incidência de doenças no pós-parto...

Tabela 4. Ocorrências clínicas observadas no pós-parto de primíparas da raça Holandesa alimentadas com diferentes fontes energéticas durante o período de transição

\begin{tabular}{lcccc}
\hline Ocorrência clínica & Controle & Megalac- E® & Soja tostada & Propileno glicol \\
\hline Número de animais & 13 & 12 & 12 & 13 \\
Retenção de placenta & 6 & 1 & 3 & - \\
Metrite & 2 & 2 & 2 & 2 \\
Deslocamento de abomaso & - & - & 2 & 2 \\
Diarreia & - & - & 1 & - \\
Doença respiratória & 3 & - & 3 & - \\
Total de ocorrências & 11 & 3 & 11 & 4 \\
\hline
\end{tabular}

MEGALAC- E®: sais de cálcio de ácidos graxos poli-insaturados.
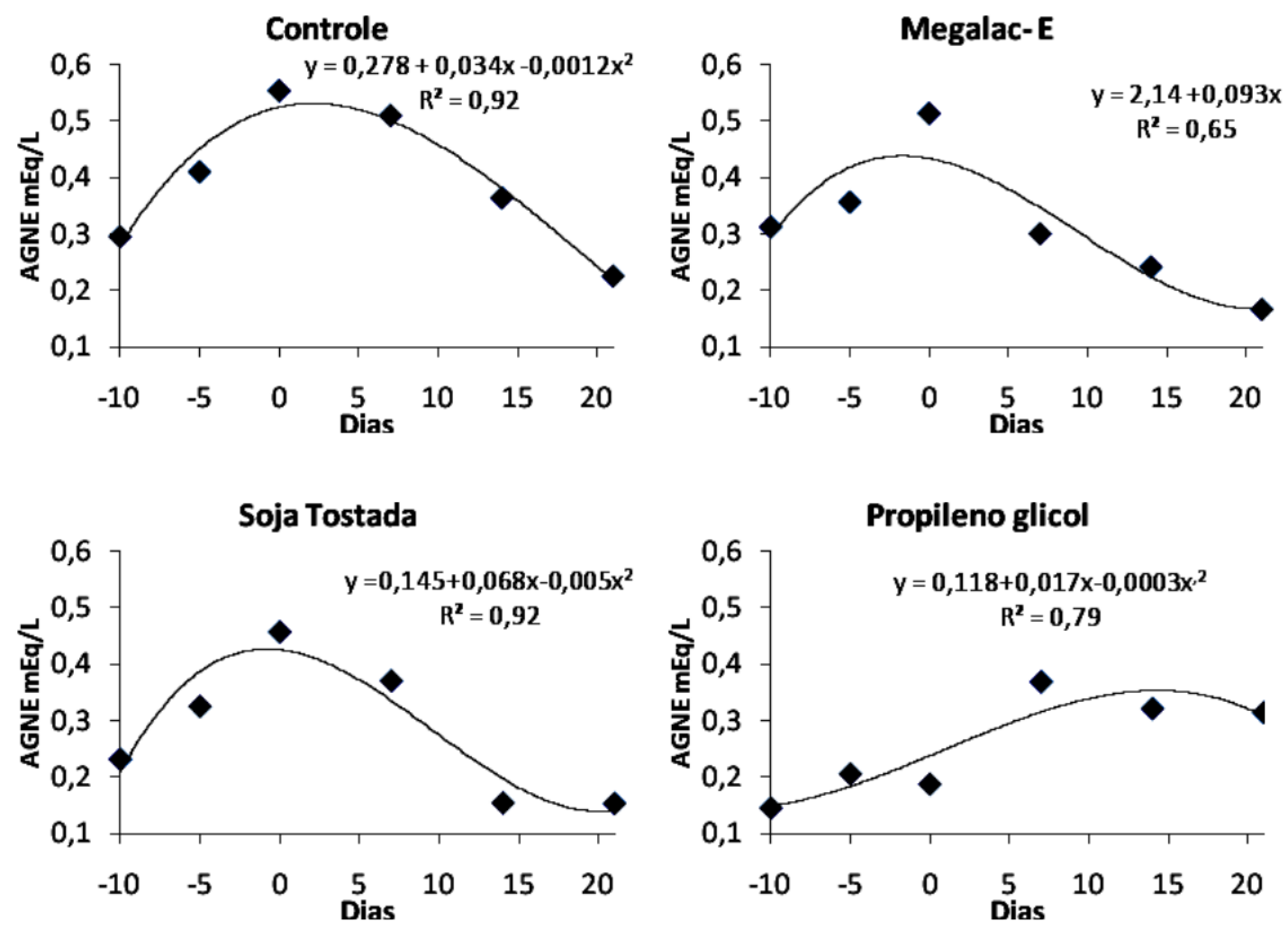

Figura 1. Concentração plasmática de ácidos graxos não esterificados (mEq/L) de primíparas da raça Holandesa alimentadas com diferentes fontes energéticas durante o período de transição.

A maior concentração de AGNE nos grupos controle, Megalac E e soja tostada ocorreu no dia do parto. Nos grupos soja tostada e Megalac E, as concentrações de AGNE declinaram a partir daí, e no grupo-controle se mantiveram elevadas até o sétimo dia pós-parto, o que demonstra que este grupo permaneceu por mais tempo em déficit energético, justificando as ocorrências clínicas verificadas. Os resultados observados para os grupos suplementados com fontes energéticas lipogênicas assemelham-se aos de outros estudos, nos quais foi utilizada a suplementação com gordura e foram observados aumentos nas concentrações de AGNE até o parto (Grum et al., 1996; Van Knegsel et al., 2007).

No grupo propileno glicol, as concentrações de AGNE apresentaram comportamento diferente, sendo observados os maiores a valores sete dias após o parto, indicando que, até este dia, o suplemento energético foi capaz de evitar grande 
mobilização corporal, mas, a partir daí, com a produção de leite em elevação, foi necessária maior mobilização corporal. Os resultados obtidos pelos vários experimentos com propileno glicol, quanto às alterações na concentração plasmática de AGNE, são controversos, existindo relatos de queda significativa (Miyoshi et al., 2001; Juchem et al., 2004) ou de manutenção de sua concentração (Grummer et al., 1994; Christensen et al., 1997). Esta variedade de resultados pode estar relacionada com a forma de fornecimento do propileno glicol, bem como com a quantidade fornecida do produto.

Quando se comparam as ocorrências clínicas entre os grupos soja tostada e Megalac E, a maior incidência de doenças no grupo soja tostada não pode ser associada aos valores de NEFA, uma vez que os dois grupos apresentaram concentrações plasmáticas muito próximas.

\section{CONCLUSÕES}

Os resultados mostram que o grupo-controle apresenta concentrações elevadas de AGNE até sete dias pós-parto, com maior incidência de afecções no pós-parto. A adição de fontes energéticas - gliconeogênicas ou lipídicas durante o período de transição foi eficiente em reduzir as concentrações de AGNE, embora os animais tenham apresentado afecções no pósparto.

\section{AGRADECIMENTOS}

Os autores agradecem à FAPEMIG, pelo auxílio financeiro para realização deste trabalho, à Fazenda Bom Jardim, por ceder as dependências e os animais, a Total Alimentos, Cooper Alfa, QGN (ARM \& HAMMER) e à NUTRON, pelo apoio.

\section{REFERÊNCIAS BIBLIOGRÁFICAS}

ARTUNDUAGA, M.A.T.; COELHO, S.G.; BORGES, A.M. et al. Primeira onda folicular e ovulação de vacas primíparas da raça Holandesa alimentadas com diferentes fontes energéticas durante o período de transição. Arq. Bras. Med. Vet. Zootec., v.62, p.116-123, 2010.
CHRISTENSEN, J.O.; GRUMMER, R.R.; RASMUSSEN, F.E. et al. Effect of method of delivery of propylene glycol on plasma metabolites of feed-restricted cattle. J. Dairy Sci., v. 80, p. 563-556, 1997.

DRACKLEY, J.K. Biology of dairy cows during the transition period: the final frontier? J. Dairy Sci., v.82, p.2259-2273, 1999.

DRACKLEY, J.K.; DANN, H.M.; DOUGLAS, G.N. et al. Physiological and pathological adaptations in dairy cows that may increase susceptibility to periparturient diseases and disorders. Ital. J.Anim. Sci., v.4, p.323-344, 2005.

GRAVET, H.O. Breeding of dairy cattle. In: Dairy cattle production. New York: Elsevier Science, 1987. p.35-76.

GOFF, J.P. Mastitis and retained placenta: relationship to bovine immunology and nutrition. Adv. Dairy Tech., v.11, p.185-192, 1999.

GRUM, D.E.; DRACKLEY, J.K.; HANSEN L.R. et al. Production, digestion, and hepatic lipid metabolism of dairy cows fed increased energy from fat or concentrate. J. Dairy Sci, v.79, p.1836-1849, 1996.

GRUMMER, R.R.; WINKLER, J.C.; BERTICS, S.J. et al. Effect of propylene glycol dosage during feed restriction on metabolites in blood of prepartum Holstein heifers. J. Dairy Sci., v.77, p.3618-3623, 1994.

GRUMMER, R.R. Impact of changes organic nutrient metabolism on feeding the transition dairy cow. J. Dairy Sci., v.73, p.2820-2833, 1995.

INGVARTSEN, K.L.; ANDERSEN, J.B. Integration of metabolism and intake regulation: A review focusing on periparturient animals. $J$. Dairy Sci., v.83, p.1573-1597, 2000.

JUCHEM, S.O.; SANTOS, F.A.; IMAIZUMI, H. et al. Production and blood parameters of Holstein cows treated prepartum with sodium monensin or propylene glycol. J. Dairy Sci., v.87, p.680-689, 2004.

KEHRLI Jr., M.E.; NONNECKE, B.J.; ROTH, J.A. Alterations in bovine lymphocyte function during the periparturient period. Am. J. Vet. Res., v.50, p.215-220, 1989. 
LeBLANC, S.J.; LESLIE, K.E.; DUFFIELD, T.F. Metabolic predictors of displaced abomasum in dairy cattle. J. Dairy Sci., v.88, p.159-170, 2005.

LeBLANC, S.J.; LISSEMORE, K.D.; KELTON, D.F. et al. Major advances in disease prevention in dairy cattle. J. Dairy Sci., v.89, p.1267-1279, 2006.

MIYOSHI, S.; PATE, J.L.; PALMIQUIST, D.L. Effects of propylene glycol drenching on energy balance, plasma glucose, plasma insulin, ovarian function and conception in dairy cows. Anim. Reprod. Sci., v. 68, p. 29-43, 2001.

NUTRITIONAL requirements of dairy cattle. Washington, DC: National Academy, 2001.
OFFICIAL methods of analysis. 13.ed. Washington, DC: AOAC, 1980.

OSPINA, P.A.; NYDAM, D.V.; STOKOL, T. et al. Evaluation of nonesterified fatty acids and $\beta$ hidroxybutirate in transition dairy cattle in the northeastern United States: Critical theresholds for prediction of clinical diseases. J. Dairy Sci., v.93, p.546-554, 2010.

VAN KNEGSEL, A.T.M.; VAN DEN BRAND, H.; DIJKSTRA, J. et al. Effect of glucogenic vs. lipogenic diets on energy balance, blood metabolites and reproduction in primiparous and multiparous dairy cows. J. Dairy Sci., v.90, p.3397-3409, 2007. 